\title{
Pfusch bei jedem zehnten Darmpolypen
}

\begin{abstract}
Auch wenn ein Adenom bei der Vorsorge entdeckt wird, ist die Gefahr noch lange nicht gebannt. Bei der Polypektomie bleibt erstaunlich häufig neoplastisches Gewebe stehen. In einer US-Studie war jede zehnte Resektion unvollständig.
\end{abstract}

Die Qualität der endoskopischen Polypektomie ist in zwei US-Kliniken prospektiv untersucht worden. Bei 269 Patienten wurden insgesamt 418 Darmpolypen mit Schlinge und Elektrokauter entfernt: 346 Polypen waren neoplastisch, davon 286 Adenome; elf Polypen waren bereits hochgradig dysplastisch und ein Polyp war ein Karzinom. 116 Polypen waren groß $(>10 \mathrm{~mm})$, die mittlere Größe lag bei $8,3 \mathrm{~mm}$. Die Hälfte der Polypen war flach, $60 \%$ waren im rechten Kolon lokalisiert.
Angestrebt war eine En-bloc-Resektion, was in den meisten Fällen (85\%) auch gelang. Sobald der Endoskopiker nach sorgfältiger makroskopischer Inspektion davon ausging, dass der Polyp komplett entfernt war, wurden von den Resektionsrändern Biopsien entnommen.

Dabei stellte sich heraus, dass von 10,1\% der neoplastischen Polypen Gewebe stehen geblieben war. Das Risiko für eine unvollständige Resektion erhöhte sich mit der Größe der Polypen: Bei einem Durchmesser von $10-20 \mathrm{~mm}$ war die Gefahr doppelt so groß wie bei Polypen von 5-9 mm. Bei sessilen serratierten Adenomen/Polypen (SSA/P) war eine inkomplette Entfernung sogar viermal wahrscheinlicher als bei anderen neoplastischen Polypen. SSA/P mit einer Größe von mindestens $10 \mathrm{~mm}$ waren zu fast $50 \%$ da- von betroffen. Sowohl die hochgradigen Dysplasien als auch das Karzinom wurden jedoch vollständig abgetragen. Wie erfolgreich die Polypektomie war, hing auch vom behandelnden Endoskopiker ab. Die individuellen Raten der inkompletten Resektionen variierten von 7 bis $23 \%$.

Die Studienautoren um Dr. Heiko Pohl vom White River Junction VA Medical Center, Vermont, gehen davon aus, dass die hohe Quote inkompletter Polypektomien eine der Ursachen für die Entstehung von Intervall-Karzinomen ist. Mithilfe spezieller bildgebender Verfahren wie Narrow-Band-Imaging oder Chromoendoskopie sollte insbesondere den Polypenrändern mehr Aufmerksamkeit gewidmet werden.

(bs)

Pohl Het al, Gastroenterol 2013, 144:74-80

\section{Gebannter Claudicatio-Schmerz}

\section{Ramipril macht pAVK-Patienten mobiler}

\begin{abstract}
PAVK-Patienten mit Claudicatio intermittens können vom ACE-Hemmer Ramipril gleich zweifach profitieren: Er senkt nicht nur das erhöhte kardiovaskuläre Risiko, sondern verbessert auch die Gehleistung.
\end{abstract}

In einer sechsmonatigen Studie verlängerten ACE-Hemmer die schmerzfreie Gehzeit bei pAVK-Patienten um 80\%, die maximale Gehzeit um 120\%, wie die Autoren um Dr. Anna Ahimastos, Melbourne, berichten. An der Studie hatten 212 Patienten mit Claudicatio intermittens teilgenommen, im Durchschnitt 66 Jahre, die bereits entsprechend ihrer Beschwerden und ihres Risikoprofils optimal behandelt wurden, allerdings bislang ohne ACEHemmer oder AT1-Antagonisten. Die eine Hälfte erhielt täglich 10 mg Ramipril, die andere Hälfte ein Placebopräparat. Nach 24 Wochen Therapie hatte sich die mittlere schmerzfreie Gehzeit von 140 auf 229 s erhöht. Im Vergleich zur Placebogruppe entsprach dies einer Steigerung um $75 \mathrm{~s}$. Auch die maximale Gehzeit nahm zu, von 234 auf $512 \mathrm{~s}$.

\section{Bessere Gehleistung unabhängig von Blutdrucksenkung}

Erwartungsgemäß war die Einnahme von Ramipril mit einer leichten Blutdrucksenkung $(-3,1 / 4,3 \mathrm{mmHg})$ verbunden. Die Zunahme der Gehleistung war jedoch unabhängig von der Blutdruckänderung. Im Placebo-Vergleich war bei den RamiprilPatienten auch eine Zunahme des Knöchel-Arm-Index zu messen - in Ruhe von 0,57 auf 0,64 , unter Belastung von 0,45 auf 0,52 . Zumindest die Steigerung des Ruhewertes war aber auf einen stärkeren Rückgang des systolischen Armdrucks zurückzuführen. Bei 111 Patienten mit verengten femoropoplitealen Arterien, darunter 50
Ramipril-Patienten, wurde auch per Doppler-Schall nach Auswirkungen der Studientherapie gesucht. Der Blutdurchfluss im Bereich der Stenose erwies sich jedoch als unverändert. Allerdings wurde ein gegenüber Placebo signifikanter Anstieg des Flusses in der A. femoralis communis proximal der Stenose festgestellt. Die mit dem ACE-Hemmer Behandelten beurteilten ihren funktionellen Status im Walking Impairment Questionnaire besser und schnitten auch im physischen Summenscore des SF-36 besser ab.

Es ist nicht ganz klar wie der ACEHemmer die Gehleistung der PAVK-Patienten verbessern kann. Man vermutet, dass außer einer Zunahme des peripheren Blutflusses auch adaptive Veränderungen in der Struktur und Funktion von Skelettmuskeln eine Rolle spielen. (bs)

Ahimastos AA et al, JAMA 2013, 309(5): 453-460 